\title{
A philosophical look at mathematics and related sciences from the beginning to the future
}

\section{Elmar Wagner}

Para citar este artículo: Wagner, E. 2022. A philosophical look at mathematics and related sciences from the beginning to the future. Ciencia Nicolaita, número 83, 200-231. DOI: https://doi.org/10.35830/cn.vi83.578

Ver material suplementario




\title{
A philosophical look at mathematics and related sciences from the beginning to the future
}

\author{
Elmar Wagner \\ Universidad Michoacana de San Nicolás de Hidalgo, Instituto de Física y Matemáticas
}

HistorIAL DEL ARTÍ́CULO

Recibido: 24 de septiembre de 2021

Aceptado: 28 de octubre de 2021

\section{RESUMEN}

Se presentan hitos históricos de las ciencias matemáticas y sus consecuencias filosóficas de manera generalmente comprensible y en parte especulativa siguiendo los logros de famosos protagonistas. De la observación del pasado, se deduce una conjetura para el futuro.

Palabras ClaVe: Filosofía de la ciencia, historia de las matemáticas, inteligencia artificial, aprendizaje de máquina.

\section{Abstract}

Landmarks in mathematical sciences and its philosophical consequences are presented in a generally understandable and partly speculative way by following the achievements of famous protagonists. From observing the past, a conjecture for the future is deduced.

KEYwORDs: Philosophy of science, history of mathematics, artificial intelligence, machine learning.

CORRESPONDENCIA DE AUTOR: elmar.wagner@umich.mx

Ciencia Nicolaita Copyright, Universidad Michoacana de San Nicolás de Hidalgo. 


\section{Introducción}

Following a subjectively selected compilation of historical landmarks, I will describe my point of view of mathematics and related sciences from the past to the present, drawing conclusions on the way and focusing not only on the results, but also on the motivations and convictions of the protagonists, in order to support a conjecture for the future. The stories that I have heard from rumors, I checked in the internet ${ }^{1.2}$ Some parts of the work were inspired by the 23 mathematical challenges of the London Institute of Mathematical Science 3 . For further discussion, everybody is welcome to contact the author.

\section{The Past}

Mathematics is one of the oldest sciences. It started with counting, as we can still see on our unfortunate number system with base ten - ten fingers - or twenty, in the case of the Maya. If our ancestors had chosen 2 as base, our kids would have to learn only $3=\frac{3 \times 2}{2}$ operations instead of $55=\frac{11 \times 10}{2}$ and we could count with our fingers up to $1023=2^{10}-1$. Our curiosity for other sciences also started at early ages, for in stance chemistry (starting a fire), biology (planting a seed to multiply it after a few months), physics (throwing a stone to hit an enemy), medicine (taking a plant to ease the pain), astronomy (observing the sun, the moon and the stars), and so on. It seems that all started with experimental science by observing and performing experiments (e.g. throwing stones repeatedly at a fixed target), all but mathematics, which used abstraction at an early stage, replacing for instance equivalent finite sets by numbers, ignoring all properties of the elements and just assigning the same word for all finite sets with the same number of elements. It was probably the first science that solved problems in a completely mental way: If I get one pot for ten apples, how many apples do I need for three pots? It also was the first science that could predict the non-immediate future of human life in non-periodic events: If I need one bag of food to survive, then my family of 5 will need five bags. Predicting the future is still a major interest of mankind, it is of such an enormous interest that fortune telling survived until our modern society.

The beginning of modern mathematics can be dated back to the flourishing of cities in Mesopotamia, India, China, and Egypt, when trading was essential and mathematics thus necessary. A similar development can be observed in the cities of the ancient civilizations of the Americas, where, apart from commerce, mathematics was used intensely in astronomical calculations. However, this report will mainly focus on the development in Eurasia because the base of modern mathematics was set there.

With the emergence of consciousness, other non-essential questions arose, for instance: Who am I? Where do I come from? Where will I go to? Unfortunately, our brain gets troubled with questions that it can't answer, so answers were given without knowing the facts. That's how religion took over and there, where religion was coupled with power, the answers to these questions became dogmas and contradictory answers were not allowed. Fear was used to manipulate people, which still works as one can see in any election battle (fear of economic loss, fear of immigrants, fear of a virus or a vaccine, etc.). As a consequence, religion turned 
into the greatest obstacle to science. Thus, a further rapid progress of science demanded a separation of believing and thinking. That was the period when philosophy arose to become the major force for acquiring human knowledge.

Interestingly, the early Greek philosophers like Thales of Miletus, Pythagoras and Euclid did not distinguish between mathematics and philosophy, mathematics was simply a part of philosophy and both disciplines posed such seemingly useless questions as "What happens after death?" or "Is the square root of 2 a rational number?" Mathematics served as an inspiration for truth (in the sense of proving statements) and deductive reasoning. From that time on, mathematics has been a permanent companion of science and philosophy. On the other hand, mathematicians started to ask their own questions without any relation to other sciences, for instance the search of Pythagorean triples, that is, three natural numbers $a, b$ and $c$ satisfying $a^{2}+b^{2}=c^{2}$. There is no immediate use of such knowledge apart from giving exercises to the students, which they can solve without a calculator.

As the mathematical knowledge and the number of mathematical texts grew, mathematics as a science was only accessible to a few specialist and highly educated people. At least, that's what happened in Ancient Greece, where the first Academies were founded. But the Greeks were conquered by the Romans who showed little esteem toward mathematics as one can still see in the cumbersome Roman number system. When the Roman Empire decayed and the church became powerful, science had to be in line with the official opinion and any discovery, which contradicted the belief system, was forbidden and sometimes even punished. Pure mathematics is not subjected to belief, so one can actually say that mathematics was among the sciences that were least affected during the dark Middle Ages within the territory of the former Western Roman Empire. Curiously it was the monks in the monasteries, i.e. the church, who continued to study mathematics. During these times, the Arabs, Chinese and Indians did better. Not without reason comes the word Algebra from an Arabic language. It is said that the Indians discovered the abstract concept of 0 , in other words, the empty set. Without 0 , mathematics could never have achieved its present potential. The Muslims brought this knowledge to Europe by trading and conquering, after all, the Spanish had plenty of contact with the Islamic world, although it was not always fortunate.

As another curiosity, mathematics found its way to astronomy and cosmology through a discipline, which is not considered a science: astrology. The belief in astrologywassostrongthatkingsandmaharajas financed their own observatories so that an astronomer could predict the position of planets and all other moving celestial bodies. Every once in a while, the same person turned into an astrologer and told the ruler some nonsense about his fortune that nobody knew, not even the astrologer.

Ironically, mathematics disguised as physics started to refute (religious) beliefs. It may be doubted that Kepler didn't know about the earth orbiting around the sun. Kepler concluded his laws of planetary motion by studying the trajectories of the planets and the sun using observations done by Tycho Brahe. One doesn't observe perfect ellipses along the trajectories unless one considers also an elliptic orbit of the earth around the sun and eliminates the movement caused by the moving earth (epicycles). When Copernicus' books were banned, he didn't de- 
fend the truth, probably life had more value for him than science. Galilei had a shot but also preferred not to test torture. Though there was a mathematical physicist and philosopher who stood by his knowledge until his cruel end by being burnt for heresy: Giordano Bruno. Any reader may take a minute and think about it if (s)he would march in such a cruel death to defend the truth. This certainly shows that there were difficult times for science in Europe.

Who could challenge such a rigid belief system? Pure mathematics couldn't because there was no mathematics in the belief system, so there was nothing in it to be challenged by mathematics. Quite the contrary, René Descartes in the 17th century and Kurt Gödel in the 20th century proved the existence of god. Both versions depend on the definition of god and Gödel's proof on some axioms ${ }^{4}$. Accepting a few axioms is something that all contemporary mathematicians have to do. If someone insists on not doing anything that involves a belief, mathematics doesn't develop its full potential. In mathematical terms, the existence of god is as true as is mathematics, one only has to accept the definitions and axioms, and to understand the proof. Gödel, who may be considered the greatest genius in logic of all times, never published his result because he feared the answer of the church and its devotees.

Descartes is also known for creating analytical geometry by linking geometry and algebra. That is another source of powerful mathematics, combining different disciplines, which were developed separately. In fact, the only reason for dividing mathematics in different disciplines is for learning, but for applying mathematics, it is much better to have all disciplines in mind and to apply and combine whatever fits best.

To answer the above question, the philosophers did it. For a second time, the philosophers had to be the vanguards of progress. Questions like "What is truth?" were difficult to prohibit and the answers often embarrassing for the church. Religion sought to mold the thinking of its adherents, but could not control all of them. So it was basically only a question of time when the church was challenged again. Fortunately, it was the humanists who promoted, together with their humanistic ideas, the interest in truth, science and progress. Even though the universe will never be completely understood, there is belief and there is evidence, and objective evidence can be crushing. On the other hand, there are things that nobody knows and everyone who says otherwise lies. For instance, Kurt Gödel's incompleteness theorems show, roughly speaking, that we will never know, nor be able to prove, that mathematics, the most basic of all sciences, is true. This raises the question if any science is true and the answer is, of course, no. Any model is only an approximation of what we call (and have to agree upon) "reality". It will keep legions of scientists busy in the future to improve the models and to find new ones.

When the philosophers changed the rules of thinking, all sciences began to flourish and so did mathematics. Physics provided such an immense number of open questions that mathematics became principally mathematical physics, apart from special interests like probability and statistics (which are good for gambling) and the omnipresent disciplines like algebra, number theory and combinatorics. Beforepublishinginjournalsbecame popular, mathematicians exchanged letters about concrete and abstract problems and competed in solving them. Sometimes they even preferred to keep formulas secret so that they could win more easily in competitions such as: Who can solve more cubic equations? Interestingly, a new level of ab- 
straction entered the game because those were the most successful who reached the highest level of abstraction. Maybe it needed such a strange character as Gerolamo Cardano, a gambler who believed in astrology, to destroy a century-old belief system of the European mathematicians, namely that there are no negative numbers. Even more, he made use of the square root of negative numbers, coined "imaginary numbers" by René Descartes, although these numbers are as real as points on a vertical axis in a 2-dimensional plane. Grasping negative numbers means understanding that there is "less than nothing" or "another direction". Adding a positive number to a negative number of equal amount yields nothing, which nowadays isn't more difficult to understand than repaying a debt or walking back to the starting point. Combining "real" and "imaginary" numbers into complex numbers requires only to step up from 1-dimensional thinking to 2-dimensional thinking, and creates the most powerful number system which only lacks the possibility to compare two numbers by their size. The use of the words "real" and "imaginary" reflects a conflict that faced the most brilliant mathematicians of this time: Is mathematics real or does it necessarily involve non-real objects created only by the human mind? At present, mathematicians study spaces of any dimension, even of infinite dimension, without ever questioning if these spaces exist in the real world.

A genuine revolution in science, often left unmentioned in general history books, occurred in the late 17th century when Newton and Leibniz discovered differential calculus. Differential calculus tells us that the values given at a certain moment of time, the local changes around us, togetherwith thechangesatthesmallestpossible (i.e. infinitesimal) time steps, determine completely the future of a dynamical system. Mathematically it says that we can reconstruct a function from the knowledge of its changes by integration, which is the inverse process of differentiation. In a contemporary language, it amounts to solving differential equations. Leibniz developed differential calculus in a notation that we still use today. Newton used his discovery to formulate the laws of mechanics, defining in that way what we call "force", and applied his theory to explain the trajectories of falling apples, cannon balls, and the planets around the sun.

It is the nature of our existence that we can measure "locally" the observables around us, and thus determine its changes, and that we postulate that the laws of nature remain the same under equal conditions. For these reasons, differential equations became the predominant equations of physics. Their use in predicting the future - one of mankind's greatest longings - was so successful that, more than a century later, physicists debated about the so-called "Laplace's demon". That is, if all forces, all particle positions and their momentums at a certain moment are known (to a demon), then the whole future of the universe will be determined (be known by the demon). Obviously, this rules out the existence of free will. This purely mechanical view of the universe ignores that our brain activity is based on thermodynamic and electrodynamic processes, and that the information processing in our brain relies on patterns, not on exact physical states. The latter, for instance, makes us see a smiling face when looking at a circle with two dots and a small arc, and the former creates a conflict with Boltzmann's second law of thermodynamics, which gives time a direction.

The simultaneous discovery of differential calculus exposed that scientific progress is 
not always driven by the quest of knowledge alone, but also by pride, fame and rivalry. Already Cardano disputed with Niccolò Fontana Tartaglia about who has to be credited for finding the solution of the cubic equation. In the matter of differential calculus, Leibniz was accused of having plagiarized from Newton. The Royal Society of London installed a committee to investigate the case. The committee ruled that - what a surprise - Newton, who coincidentally was the president of the Royal Society at that time, had first invented calculus. Even geniuses like Newton are humans after all.

In the following decades, the Bernoulli family dominated the research in mathematics and physics, perhaps the most influential family in science. They were excellent masters in solving very specific problems analytically, often challenging each other. Such calculation skills are hard to find nowadays since it is now faster and easier to get an approximate solution by a computer. Geniuses and humans like Newton, their enormous output of results was fueled by rivalry and jealousy, even among family members.

From the Bernoulli family descended the greatest mathematician of the 18th century, not as a family member but as a student: Leonard Euler. He made groundbreaking contributions to almost all branches of contemporary mathematics at that time. For instances, with his famous problem to find a way in Königsberg which crosses each of its seven bridges only once, he initiated graph theory, and with the observation of the invariance of the number $\chi:=$ \#vertices - \#edges + \#faces for Platonic solids, he started the study of topological invariants even before the concept of a topological space had been defined. His analytical reasoning for problem solving established the fundamentals of modern mathematics. Much of his mathe- matical language is still in use today and, as known from psychology and philosophy, language influences our thinking. "Die Geisel", female in German, is called "el rehén" in Spanish, a male word. This will influence how German and Spanish people think about a hostage even if they describe the meaning with the same words.

Euler was the most prolific mathematician of his time, no serious disputes with his fellow mathematicians are known and his dedication to mathematics was so strong that not even losing his eyesight lowered his productivity. It is reassuring to conclude that the most influential mathematicians are not necessarily driven by rivalry and jealousy, but a genuine interest in the progress of mathematics.

Euler is credited for the potentially most beautiful formula in mathematics:

$$
1+e^{i \pi}=0
$$

This formula contains two indispensable transcendental constants: the number $\pi$, which is ubiquitous for describing anything related to circles, spheres and rotations, and Euler's number e, the natural base for exponential functions. The effects of exponential growth can be seen for instance in the spread of a virus at the beginning of a pandemic, and it also explains why evolution works and can be so diverse. Evolution is not just "survival of the fittest", it is first and foremost diversity in huge numbers before evolutionary pressure drives the average in one or another of the countless directions. To put it plainly, human intelligence is a result of exponential growth.

However, Euler's formula has a small imperfection. Unfortunately, Euler denoted by $\pi$ the ratio of a circle's circumference to its diameter, but a circle is conceptually better characterized by choosing the center and a single point on its circumference, so the ra- 
dius is a more natural parameter. In particular, a full rotation corresponds $\Pi:=2 \pi$. With $\Pi$ as the characteristic number of a circle, Euler's formula becomes

$$
1+e^{\mathrm{i} \Pi / 2}=0
$$

Now, apart from the famous transcendental constants, the formula contains the basic numbers $0,1, \mathrm{i}:=\sqrt{-1}$ and also 2 , thus all we need for our most complete number system. With 2 as a base, we can express any real number as a combination of 0 s and $1 \mathrm{~s}$, and adding another direction by $\mathrm{i}=\sqrt{ }(-1)$, we obtain the powerful complex number system, where any polynomial equation has a solution and no point of the plane is missing. It seems that mathemtics is a very conservative science, we will never see in our life the practical redefinition of

$$
\Pi:=2 \pi=\text { one full rotation }=\text { one period }
$$$$
\text { of sine and cosine, }
$$

and we will never teach our kids in kindergarten the simple binary number system although all our computers use it. Humans don't like changes of what they got used to.

There is one thing that Euler seemed to have understood thoroughly, but never defined precisely: infinity. The Greeks discussed already the infinite large, the infinite small was used by Newton and Leibniz and their successors (for instance by Euler), but the rigorous handling of the infinite started with Cauchy, a trained engineer with a strong interest in mathematics and the founder of complex analysis. There are so many theorems named after Cauchy that no graduated math student will ever forget his name. Undergraduate students become so familiar with Cauchy's definition of the infinite small that, when suddenly woken up at night and asked "How big is epsilon?", they will almost certainly answer "It's small!" (it can be smaller than any positive real number). Cauchy may have produced even more theorems if he wouldn't have had such strong conservative political opinions that complicated his life. Sometimes science is an obstacle for (despotic) politics, sometimes political beliefs are an obstacle for science.

Without the infinite, mathematics would be a science of rules and logic but without tools. With the correct understanding of the infinite, the infinitely small and the infinitely large, progress in mathematics exploded in the second half of the 19th century, again with an enormous input from physics. Old problems like Laplace's, Poisson's, wave and heat equations could be studied in a new light and on a solid foundation by the use of Fourier's trigonometric series and integral transformations, later called harmonic analysis. Analysis separated from algebra, and new areas like topology and set theory evolved at a fast pace. Interestingly, until the 20th century, mathematicians were not able to state properly what the notion set means. As the mathematical knowledge grew, specialization and division into different disciplines increased. I will return to this later.

Much of the rigorous groundbreaking work was done in the first half of the 19th century by Carl Friedrich Gauss, who may be considered as the greatest mathematician of all time, although he had an obscure spartan writing and teaching style that was difficult to understand and much hated by students. Not always is the best scientist also the best teacher. Famous already during lifetime, his pursuit of knowledge was not driven by fame or rivalry but the desire of understanding a (mathematical) problem deeply and completely. An indication of this is that Gauss left many pioneering discoveries unpublished because he considered them incomplete although the 
latter led to priority disputes.

One of these disputes concerned non-Euclidean geometry. Gauss claimed to have discovered non-Euclidean geometry before Lobachevsky ${ }^{6}$ and Bolyai ${ }^{7}$ but never published any result in his lifetime. Maybe he considered his treatise incomplete, maybe he feared the reaction of his colleagues because the discovery of non-Euclidean geometry challenged the common perception that $\mathrm{Eu}-$ clidean space is the only valid geometry. It also questioned the necessity and validity of axioms (e.g. Euclid's parallel postulate), and raised concerns that human perception leads to unnecessary postulates or that missing postulates lead to mathematical theories that cannot be considered true. Non-Euclidean geometries are intimately related to curvature, an intrinsic measure of how a spacebends and which is independent from an embedding into Euclidean space. Gauss described the curvature of surfaces in his famous Theorema egregium ${ }^{8}$, which implies for instance that one cannot draw a map of the earth on a sheet of paper without distortion. The Gauss-Bonnet theorem, which relates the integral of Gaussian curvature to the Euler characteristic $\chi$ defined above, can be considered as a precursor of one of the most celebrated theorems of the 20th century: the Atiyah-Singer index theorem ${ }^{9}$. Gauss' student Bernhard Riemann extended the notion of intrinsic curvature to spaces of any dimension ${ }^{10}$ and established in this way the geometric foundations of Einstein's general relativity ${ }^{11}$. From the simple consideration that it is more likely that the universe is finite, it becomes more natural to view space-time as curved and not as Euclidean.

Another surprise in those times was the most famous "no-go theorem" in mathematics, arguably one of most difficult theorems that undergraduates have to learn and seemingly too difficult for some of the best mathematicians in the first half of the 19th century because it took more than a decade to acknowledge that Évariste Galois solved a mathematical puzzle that had defied a solution for centuries ${ }^{12}$. Almost 300 years before, Cardano published solutions of polynomial equations of degree 3 and 4 (lower degrees are easier). After many failed attempts, doubts emerged that there exists a formula for equations of degree 5 and higher, involving only algebraic operations and (higher-order) roots. It should be mentioned that Niels Henrik Abel ${ }^{13}$ proved the non-existence of a general formula 6 years before Galois submitted his work, but Galois created a whole new theory that allowed to decide when such a formula exists. Both were geniuses, because both solved hard problems at an early age. Sadly, both have in common that they died very young in their twenties. It is difficult to imagine what these brilliant minds would have contributed to mathematics in a normal lifespan. Abel died poor from tuberculosis working hard until his end. Galois had a rebellious mind, which often brought him into trouble and ultimately into a fatal duel, presumably because of a love affair. Foreshadowing his fate, he used the last night to write letters that preserved his mathematical legacy but not his life. Whatever his thoughts might have been in those hours, maybe it needed such a rebellious mind to think differently than the renowned mathematicians of that time.

Remarkably, Galois and Abel initiated by their work the breakthrough of a mathe-matical structure that has always and nearly unnoticed been around: group theory. Groups are the simplest structures where two elements can be combined in an asso- ciative way to a third one and where equations like $a$ o $x=\mathrm{b}$ can be solved for unknown $x$. For being so simple

\footnotetext{
${ }^{6}$ (Lobachevsky 1837) $\quad{ }^{7}$ (Bolyai 1832) $\quad{ }^{8}$ (Gauss 1828) $\quad{ }^{9}$ (Atiyah and Singer 1968) $\quad{ }^{10}$ (Riemann 1868)

12 (Galois 1846) $\quad{ }^{13}$ (Abel 1824)
} 
and allowing to solve equations, groups are used nowadays to classify more complicated objects like manifolds (spheres, tori, projective spaces, etc.). Groups and symmetries are essentially the same thing, groups always act as symmetries (e.g. on itself) and a (complete) set of symmetry transformations always forms a group. Symmetries were already used by our ancestors before becoming humans, a perfectly symmetric fruit looks more beautiful than an asymmetric one and sends the correct message of having had better growing conditions and thus of being more nutritious. In the same vein, a mirrorsymmetric mating partner looks healthier than an asymmetric one. What we call the beauty of a flower is a competition of attracting insects for reproduction, even such primitive brains like the one of an insect can perceive symmetry. The great mathematical physicist Hermann Weyl went so far to state that beauty is symmetry ${ }^{14}$. Whereas symmetry always signifies beauty, psychologists found out that a slight deviation from perfection attracts more attention, for instance in mating habits. It is the variation of genes that pushes evolution. Nevertheless, I would go so far to state that groups are the most beautiful objects in mathematics. Groups are as beautiful as a perfect circle (the group $\mathrm{U}(1)$ ), as a perfectly round 3-sphere if you can imagine a sphere in 4 dimensions (the group $\mathrm{SU}(2)$ ), or a perfect 2 -sphere, which is not a group but on which acts the group $\mathrm{O}(3)$ of orthogonal isometries of $\mathbb{R}^{3}$ (it is a homogeneous space).

Groups are today a principal tool in mathematics. They allow to make calculations only in a single point (for instance the Gauss curvature of the 2-sphere) and then to know what happens at any other place by moving the point around the space using an (isometric) group action. It is totally astonishing that their systematic study started less than 200 years ago. Galois used permutation groups to study the symmetries of the roots of polynomials. Felix Klein was so exited about groups that he started the Erlangen program in 1872 , a program of classifying geometries by group actions. Sophus Lie combined group theory and differential calculus to eliminate unnecessary parameters in differential equations, much in the same way as Kepler's laws are used to reduce the parameter space of planetary orbits, and thereby created the vast field of Lie groups and Lie algebras. The classifications of semi-simple complex Lie algebras by Élie Cartan ${ }^{15}$ in his PhD thesis, and of all finite simple groups by many contributors, were major achievements in the last 130 years. Here, "simple" means "building blocks" of more complicated structures. That's the way mathematicians think, once they have discovered an important structure, they want to know all possible objects (up to equivalence) and that will keep them busy for centuries. As alluded above, understanding groups is a good starting point for such programs.

In physics, the standard model is based on the Lie groups U(1), SU(2) and SU(3). Mathematicians call this model Yang-Mills theory and can win a one million dollar prize from Clay Institute of Mathematics 16 if they prove its last mysteries like the mass gap. Einstein's special relativity 17 is essentially the theory of isometrical group actions on Minkowski space. This was already known to Lorentz, who observed that Maxwell's equations of electrodynamics are invariant under these transformations, but it is to due Einstein's $E=m c^{2}$ draw the right conclusions like the twin paradox, which isn't a paradox at all, it just violates human's perception of an absolute time. The twin paradox allows particles to reach our planet, which have a lifetime less than the time that light needs to travel from the sun to the earth. 
With his unique thinking and the shattering of human's perception of an absolute time, Einstein became the first pop icon of science. Young folks scribbled the formula on city walls, possibly without understanding it or because of the joy of understanding it. Indeed, understanding something awesome can be quite exiting. A paparazzi photo of him with his tongue sticking out went viral before social media existed. Legends were constructed around him like the one that he failed mathematics at high school. Probably a German saw his school certificate without knowing that the Swiss used an opposite number system compared to Germany, where the lowest number 1 corresponds to the best mark. These details don't matter if ordinary people can find delight in failures of pop stars and reassure themselves that geniuses are just like them, which they are not.

Having reached Einstein, it would be much too much to mention all geniuses of mathematics and mathematical physics in this exposition, so I will have to restrict myself to just a few ones. First of all, it is rather unavoidable to mention two senior contemporaries of Einstein: Henri Poincaré and $\mathrm{Da}-$ vid Hilbert. What they have in common is that both can be considered the last universal geniuses of mathematics in the sense that they contributed profoundly to almost all the important mathematical disciplines of their time and established new ones. From this perspective, they were on an equal footing with Euler and Gauss. After that, mathematics became too diverse to be grasped by a single human mind.

Poincaré got famous for many discoveries, and also for a problem that he didn't solve but only conjectured, the famous Poincaré conjecture. Roughly speaking, it says that the usual topological invariants ("number of holes") are enough to characterize a sphere, where the

${ }^{18}$ (Poincaré 1890) 3-sphere resisted a proof for a long time. For mathematicians, it is a very important question because if it had a negative answer, they would have to look for more topological invariants making the classification of topological spaces more difficult. The Clay Institute of Mathematics considered this problem so important that they included it into their seven Millennium Prize Problems and offered one million dollars for a solution.

In physics, Poincaré discovered that the Lorentz transformations form a group that leaves Maxwell's equations of classical electromagnetism covariant, i.e., they transform in the right way. (Having mentioned the "most beautiful equation of mathematics", I vote for calling Maxwell's equations the most beautiful equations of physics.) Thus, Poincaré had all pieces of special relativity together but there was one piece too much rather than missing, he couldn't abandon his belief in an aether theory, that is, a space-filling substance for the transmission of light. It needed a radical mind like Einstein's to draw the right conclusions. In this example we can see again how belief disturbs the progress of science, even more if the believer is a well-respected scientist. Being an intuitionist, Poincaré was also a master of "as easily seen", a bad habit of mathematicians to cut lengthy and possibly difficult proofs down by disparaging the reader's knowledge: "Can't you see? It's easy!" For math students, it may be the most annoying phrase.

These remarks do not downgrade Poincarés ethical standards. He participated in a competition for a prize awarded by the Swedish king about the stability of the planetary system. Poincaré reduced the problem to a three-body problem with one of small mass ${ }^{18}$. Yet his submission was too difficult to understand even for the jury although nobody had doubts about the quality of Poincarés work. Poincaré was awarded the prize 
but when an editor asked some critical questions, he found a mistake in his treatise, which flipped the result from stability to a possible instability. He was asked to correct the error and to buy back all published copies, which he did, although it was quite a bit more expensive than the prize money. So truth, or his reputation, mattered more to him than money. By correcting his error, he initiated a new mathematical discipline called chaos theory, commonly described as the "butterfly effect". It may be noted that even highly qualified referees cannot guarantee the correctness of a difficult paper. Let's hope that our main theorems don't contain mistakes!

Besides, Poincarés work casts doubts on the Laplace demon. What happens if the demon is slightly wrong about the initial conditions? Then the butterfly effect tells us that the nonlinearity of the problem can cause huge changes in the outcome, which makes the prediction of the future an extremely difficult task.

Pretty much in the same way as Poincarés work relates to Einstein's special relativity, Hilbert's work relates to Einstein's general relativity. Almost simultaneously with Einstein, he proposed an action principle leading to curved space-time ${ }^{19}$. Being a role model of a scientist, Hilbert never engaged in a priority dispute and voluntarily granted all credits for the discovery of general relativity to Einstein. One might speculate that Hilbert was famous enough and didn't need more fame, but so was Newton when he disputed with Leibniz.

There is a huge difference between Poincarés and Hilbert's style of doing mathematics. Poincaré focused on insights and comprehension in an intuitive way. In his preference of intuition over logic, he appears to be rather a physicist than a mathematician. Hilbert, on the other hand, pursued an axiomatization and rigorous foundation of mathematics on

\footnotetext{
${ }^{19}$ (Hilbert 1915) $\quad{ }^{20}$ (Hilbert 1900)
}

the basis of logic. A simple paradox, known as Russell's paradox, plunged mathematics into crisis. The paradox asks for the set of all sets that don't contain themselves and showed that a naïve understanding of sets is not enough for mathematics. To solve the paradox, one needs a rigorous definition of the concept set. Some mathematicians like Brouwer decided not to use concepts that cannot be constructed in a proper way, and that a mathematical statement is true as long as it doesn't create a contradiction. Therefore, nothing that creates a contradiction should be constructed, like the set of all sets that don't contain themselves.

Hilbert's approach was to set up a complete system of axioms and rules, which is free of contradictions and from which all results in mathematics can be deduced. Gödel's incompleteness theorem mentioned above showed that Hilbert's goal can never be achieved. Nowadays most mathematicians accept Zermelo-Fraenkel set theory with the axiom of choice. Nevertheless, Hilbert's attempt had such an enormous impact that his style prevails in modern mathematics (and Poincarés style still in physics).

Hilbert was a genius with such an astonishing comprehension of mathematics that he shaped the mathematics of the last century with a single talk at the International Congress of Mathematicians in 1900 and the posterior publication about it ${ }^{20}$. Hilbert proposed 23 problems covering a wide range of topics in which he clearly prioritized his axiomatic approach. Some of these problems are still unresolved. One of these problems, the Riemann hypothesis, even made it into the seven Millennium Prize Problems with a prize money of one million dollars. Never again has a single mathematician had such an exhaustive vision about mathematics.

A genius with an understanding of a wide range of mathematical and physical 
disciplines (almost all, but mathematics developed during his lifetime in too many directions to be followed by a single human mind at top level) was John von Neumann. His impact on mathematics is comparable with Poincaré and Hilbert in terms of creating and boosting new theories. John von Neumann participated in two scientific revolutions that haven't been mentioned yet: statistical physics and quantum mechanics, both developed around the turn of the twentieth century. John von Neumann contributed substantially to the mathematical foundations of these theories. For both theories, he used a reformulation in terms of operators on a Hilbert space. As the name suggests, Hilbert started to study these objects and even used the name spectrum for a set of (real) complex numbers that characterize (self-adjoint) operators on a Hilbert space. It is not known if Hilbert was aware that these numbers would later become identified with the outcome of possible physical measurements like the spectrum of light.

Statistical mechanics, which explains the existence of irreversible processes, was developed by Ludwig Boltzmann 21. It roughly says that it is not impossible that a system returns to its initial state but usually the probability is extremely small. For this, Boltzmann introduced the concept of entropy that "measures" the disorder, and isolated systems tend to increase their disorder. To put it simply, it is more likely to find the system in a state that is more likely. Statistical mechanics also provides a mathematical definition of temperature, which is quite different from just feeling cold or hot; and a direction of time, it is the direction in which the entropy increases, and statistically the entropy "always" increases. Of course, the entropy can decrease locally, which means that the order increases, otherwise our brain could not exist, but it is at the cost of global disorder, that's why we need to supply our brain with energy to keep it functioning. Small particles like electrons and protons prefer order, an electron enjoys being captured by a proton. It is a more stable state of lower energy and stable states last longer. Provided that certain states are longer stable at the cost of an increase in total entropy, it will be even more favorable for the second law of thermodynamics. As long as specific organic compounds, cells, organisms, brains, etc. owe their existence to an energy flux that increases the total entropy, there is no contradiction to the second lawof thermodynamics. For the existence of life, it needs a driving force, the increase of stable states by replication, again by an energy exchange that rises the total entropy. Replication, or let's say reproduction, summarizes pretty well the purpose of life. If you need more purposes, you have to give your life some more.

The existence of (intelligent) life is no contradiction to the second law of thermodynamics, it started with a fluctuation within an inconceivable huge number of possibilities and evolved as a self-reinforcing process. The mathematics to deal with such huge numbers of possibilities, with chaos, nonlinearity, emergent structures and internal self-reinforcing processes, are studied under the name complex systems. It is still an emergent discipline and a promising research area for winning a Nobel Prize since the evolution of life, the global economy and the behavior of societies can be modeled by complex systems. Life is not a mystery, the mathematics behind life is the mystery.

Statistical mechanics was a true revolution. Scientists of Boltzmann's era weren't even sure whether atoms existed. After the success of Newton's mechanics, physicists began to believe in Newton's mechanics like in a dogma. However, it is invariant under time reversal, whereas Boltzmann showed the existence of 
a direction of time. The problem seems not to have been that this was too difficult to understand, the dilemma was the obvious fact that Boltzmann's law couldn't be deduced from Newton's mechanics alone, so Newton's mechanics could not be considered complete. This provoked a major crisis in physics, much worse than the crisis in mathematics when logicians understood that mathematics needs to be founded on axioms but can never be proven to be true. For the understanding of the physical world, the need of mathematical

axioms didn't mean much as long as the models work (for instance quantum field theory is still not founded on rigorous mathematics), but Boltzmann showed that mankind was far from understanding everything because if there is one more law that nobody knew, then nobody knows how many laws are missing. Even Nobel Prize winner Ostwald (in chemistry!) refused to accept the theory of atoms. Unfortunately the criticism from some colleagues may have contributed to Boltz- mann's deteriorating mental health and to his tragic death, he did not live to see the victory of his ideas. Statistically, geniuses have mental health issues as often as ordinary people unless otherwise proven.

John von Neumann gave a novel description of entropy by combining it with the other mentioned scientific revolution: quantum mechanics. Before quantum mechanics, Newton's mechanics described the dynamics of massive particles and Maxwell's equation described electromagnetic waves. In particular, there was an obvious difference between particles and waves. However, the newly discovered photoelectric effect, i.e. the emission of electrons caused by light of certain frequencies, could not be explained by a wave theory. As Boltzmann suggested years before, Max Planck hypothesized that energy states can be discrete and resolved in that way the diver22 (Planck 1901)

${ }^{24}$ (de Broglie 1925) ved in that way the diver- he has

${ }^{25}$ (Schrödinger 1926) gence problem in computing the spectrum of black-body radiation ${ }^{22}$. Einstein, in his unique way, postulated that the quantization of all electromagnetic radiation is not just a mere computational tool but an actual fact of nature, i.e. light travels in particle-like packages, and explained thus successfully the photoelectric effect ${ }^{23}$. For this discovery, Einstein was awarded the Nobel Prize in Physics - and not for his revolutionary relativity theory. Anyway, he didn't need two Nobel Prizes to be remembered as a genius.

Louis de Broglie picked up the idea of wave-particle duality in his $\mathrm{PhD}$ thesis, postulating that all particles have wave-like properties ${ }^{24}$. His hypothesis was later confirmed in diffraction experiments with electrons. For his PhD thesis, de Broglie won the Nobel Prize.

There aren't many PhD theses worth a Nobel Prize, and I am afraid that some universities would have rejected such a daring thesis as nonsense. For physicists, it was quite a challenging hypothesis that matter behaves as particles and waves, but for mathematicians, it should be of less concern, particles and waves are just two mathematical models, i.e. theoretical approximations, for the same objects. Anyway, if some student strives to win a Nobel Prize for her/his PhD thesis, (s)he may try to find a unifying picture for the so-called wave-particle duality. My naïve suggestion would be to study sections of vector bundles.

These sections behave like wave function, may look very harmonic on nice spaces, come with a discrete set of topological (quantum) numbers, may have a singularity (a kind of vortex) at a single point (that could be blown up to a manifold), and the idea fits into a previously mentioned one million dollar prize problem from the Clay Institute of Mathematics, so it is actually not a completely new proposal. Of course, to win the Nobel Prize, (s) he has to make verifiable predictions that are 
and are studying its properties and solutions until now. In 1925, the physicist Werner Heisenberg, who was rudely rejected to study mathematics at the University of $\mathrm{Mu}$ nich for his interest in mathematical physics, spent some time in scientific isolation at an island in the North Sea to recover from an allergic rhinitis. Having had time to think about physics, he tried to find a formula to calculate the spectral lines of hydrogen. To ma tch observable results, he departed from a new approach, considering differences (instead of absolute values) of frequencies and intensities of spectral lines. Summing these differences up in a certain way, he obtained a correct result. When he presented his formula to Max Born (who had studied mathematics under Hilbert and Klein), Born realized that this certain way of summation was nothing else than matrix multiplication. Together (with Pascual Jordan) they formulated the so-called matrix mechanics ${ }^{26}$ in contrast to Schrödinger's wave mechanics. These are apparently quite different theories. In Schrödinger's theory, the wave functions evolve with time; in Heisenberg's theory, the matrix operators depend on time. For the sake of completeness, all scientists mentioned in this paragraph and not written in parenthesis won the Nobel Prize in Physics.

The fact that matrices usually don't commute had a surprising implication: the corresponding observables cannot be measured (= known) simultaneously with arbitrarily high accuracy. This is called Heisenberg's uncertainty principle. Heisenberg's uncertainty principle for position and momentum together with the probability interpretation of Schrödinger's wave mechanics were the final blow for the Laplace demon, although Einstein wanted to save the demon with a hidden variable theory. It is interesting to note that Einstein, who revolutionized phy- sics and shattered preconceived notions by radical interpretations of the physical reality, became a victim of his own beliefs and objected the probability interpretation of quantum mechanics without proper evidence (he only presented his famous thought experiments). A scientist, no matter how successful, should never stick to personal beliefs, but unconsciously will always do so.

It's curious, I don't know anybody who believes that Riemann's hypothesis is wrong, and I don't know anybody who states that it is a fact.

The demise of determinism opens the door to free will. But what is free will? If you think that either there exists free will or it doesn't, then you are probably mistaken. It is not that simple, at least if you believe in causality. Did you ever ask yourself if a computer with a couple of simple programs running on it has free will? If you did, then you probably have answered yourself "no", it has no free will, like any other machine running on pre-installed programs. The most primitive life-forms were programmed by Mother Nature to do the "right" thing - Escape! From the enemy. Eat! What is nourishing. Sleep! In a safe place. because Mother Nature punished the opposite behavior (the "wrong" thing) with extinction, that's natural selection. These life-forms have only instincts because they were programmed to do always the same thing in a specific situation.

But what happened, when there were two stimuli at the same time? If both responses at the same time aren't possible, there is a need to decide. At first, the chance to do one or the other action may be exactly 50:50, i.e. 0.5 in terms of a probability measure. In other words, to make randomly a decision between two choices with the same probability requires exactly 0 free will. If you absolutely don't care about coffee or tea, no matter what you 
had last time or smell first, your decision doesn't need any free will. You may even have made a decision before you know it (Libet experiment). However if you absolutely don't care about toxic and non-toxic berries, then Mother Nature may punish you with extinction. To increase the chances for the survivors to survive, Mother Nature will have to store information in them, for instance by bitter and sweet taste (fear/pleasure, pain/joy, etc.), so that toxic berries get rejected by instinct. If you nevertheless eat the bitter berries contrary to your instinct and without a need, for instance to test if they are toxic, then you execute free will, very likely not total free will because there is causality, and you probably had a motivation (i.e. expected benefit) to do so. Instinct evolved step by step by evolution, and so did its opposite: "free" will together with consciousness. Before the emergence conscious-ness, emotions ruled our behavior, and for some people, they still do.

This raises the question of what is consciousness. As our brain works on the basis of electromagnetic, thermodynamical and chemical processes, I have already answered the question. Mathematically, consciousness is nothing else then information processing of statistical and self-organizing complex systems together with feedback and control. To keep a long story short, the mathematical foundations for a model approximating consciousness do already exist, but they are too complex to give an answer now, there will be a lot of research about it in the future.

To each decision, there can be assigned a number between -1 and 1 that represents the amount of free will. It is roughly given by $1-2 \mathrm{p}$, where $\mathrm{p}$ denotes the probability to do it by instinct. So -1 means pure instinct like pulling the hand away when touching burning coal, and 0 free will means a completely random decision between two options with the same probability. A free will of 1 is difficult to achieve because there is causality and there are motivations based on previous experiences, involuntary emotions or learnt instincts. Being voluntarily crucified to live up to one's own words regardless of the torture that accompanies the death by crucifixion is pretty close to a free will of 1 , as is starving oneself nearly to death on the quest of truth.

However, how we experience free will is a different question. Now you may ask yourself: Where does "free" will come from? May I ask you a different question? Did you ever read this text before, I mean exactly the same text written by another person? Mind can create new thoughts by combining available information in novel ways. The more information is available and linked in the brain, the more combi-nations are possible, that's why nobody can become a genius without learning. Thoughts allow decision-making based on experiences and knowledge, and to make a decision opposite to your instinct, you must have had thought about it. Furthermore, it requires an understanding of past, present, future and causality. "If it rains tomorrow, I will stay at home." If $=$ a conditional clause, it rains $=\mathrm{a}$ possible future event, stay at home = a decision based on emotions (I don't like rain) or past experiences (it will be too cold for swimming). "Free" will manifests in decisions made in the past for events that occur in the future. Immediate spontaneous reactions are not free will, they are called reflexes.

Let's return to quantum mechanics. Having two reasonably correct theories for the same phenomena - Schrödinger's partial differential equations and Heisenberg's matrix mechanics - can have two causes. Either both are different approximations of a not yet completely understood physical principle, or both are the same theory in different languages. That's where John von Neumann entered the 
That's where John von Neumann entered the game. He used a theorem by Stone and proved the uniqueness of Schrödinger's operators ${ }^{27}$, which shows that Heisenberg's matrices and Schrödinger's operators are essentially the same objects in different descriptions. What I find astonishing is not the proof, what astonishes me is that he had the insight to prove that two utterly different looking theories are the same.

When von Neumann gave the correct mathematical description of Heisenberg's matrices as operators on a Hilbert space, the physicists got a little scared because some (invented) matrices are not defined on any state of a Hilbert space. Proving the required condition of self-adjointness can be a difficult task that physicists gladly leave to the mathematicians. John von Neumann laid the mathematical foundations of quantum mechanics in a book of the same name ${ }^{28}$. It is said that, when he wanted to publish a long article in a Springer journal, the editor feared a loss of profit since the space could be used for several other papers, so von Neumann had to promise to write a book for the same publisher. If the story is true, the editor was a clever businessman because the book Mathematical Foundations of Quantum Mechanics became a standard reference for functional analysis, quantum mechanics and quantum thermodynamics.

During the horrible Nazi period, von Neumann's family emigrated to the United States, as many European scientists of Jewish heritage or with opposite political views did, for instance Albert Einstein. So many scientists left Europe that, when Hilbert was asked if the Mathematical Institute in Göttingen had suffered by the departure of the Jews and their friends, he replied: "It doesn't exist anymore." For the United States, it was the beginning of a time of flourishing in science and technology by attracting the smartest brains (which the US still do).

When it became clear that nuclear fission could be used to construct powerful bombs, a letter signed by the pacifist Albert Einstein was sent to the US president that warned against a possible German atomic bomb and indirectly suggested to build one before the Germans did. As a response, the US government launched the top-secret Manhattan Project aimed at developing the first nuclear weapon. Among the mathematical experts joining the project were Stanislaw Ulam and John von Neumann. There may be many reasons for joining a project developing a weapon of mass destruction, for instance patriotism, money, fear of the enemy, and also scientific curiosity. Whatever the reason might be, it raises the ethical question whether scientists should participate in military projects aimed at killing people. Being one of the founders of mathematical game theory, von Neumann had his own theory about peace. If two or more players can never win but definitely destroy the other, no one will ever dare to start the game. He has been right so far, but he didn't consider the psychology of a mad "Führer" who starts a war that he cannot win, or a narcissistic leader who is a sore loser and prefers total destruction of a proclaimed enemy over giving up power. Please check the psychology of the candidates before voting, they may ignore all human knowledge gained by game theory.

To perform computations for the creation of the deadliest weapon of mass destruction, John von Neumann found out that it was faster to program a computer than to do the computations manually. As there was the computer ENIAC in the US around, John von Neumann wrote computer programs, algorithms and described a computer architecture. Together with Ulam, von Neumann de- 
ped a stochastic simulation, called Monte Carlo method, which is still in use today. His novel contributions made him one of the founding fathers of computer science and Artificial Intelligence. However, his involvement in the creation of nuclear weapons had a price. His curiosity let him not only compute models, but also participate actively in the construction. He didn't become very old, he died from cancer.

Perhaps I should mention another famous genius among the renowned scientist participating in the Manhattan Project that sparked a major boost in nuclear physics: 25 years old Richard Feynman who later was awarded a Nobel Prize in Physics for his famous diagrams that bear his name ${ }^{29}$. Feynman diagrams are a primary computational tool to symbolize heavy computations in quantum field theory. Some mathematicians are used to symbolic computations although others reject them as a valid mathematical proof. For instance, the operations of a braid group can be visualized by braided strings so that everybody can see immediately the rules without even knowing what a group is and how the relations are defined, one just has to pull the strings and to unravel them, thus applying in each step the correct mathematical rule.

Feynman diagrams are such symbolic rules that tell physicists what to do without the need of a rigorous mathematical proof. Just a word of warning, these diagrams depict mathematical computations and not the reality (they contain "virtual particles" faster than light) - the outcome of the computations tells us something about the reality. In fact, these diagrams were at first rejected by Feynman's senior colleagues, although their inventor could perform computations on a blackboard in several minutes, which would take hours or days to verify. They describe a perturbative method to compute something that is

\footnotetext{
29 (Feynman 1949)
}

30 (Dyson 1949)

31 (Dyson 1952) mathematically given by a series. It was Freeman Dyson ${ }^{30}$, who made mathematical sense out of these diagrams, and who furthermore showed that the series actually diverges ${ }^{31}$, so he also proved that, mathematically, the series doesn't make sense at all.

Feynman was an unconventional physicist and an intuitionist, so he was pretty far from rigorous mathematics. From all math- ematicians and physicists mentioned in this paper, he may be the one who is furthest away from pure mathemtics. As his Manhattan Project colleague John von Neumann, Feynman died from cancer.

Having mentioned von Neumann's role in computing, there was another extraordinary mathematician who can be considered a founding father of computer science, the one who helped to crack the Enigma code of the Nazis: Alan Turing. Contrary to the development of an atomic bomb, his involvement in World War II may have saved many lives by deciphering top secret Nazi messages. It seems that he was not only the first person who designed an abstract computer machine, but also the first one who had a clear vision for Artificial Intelligence ${ }^{32}$. Around 70 years ago, he introduced the so-called Turing test, which does not ask if a machine is intelligent but rather if a machine can perform tasks that cannot be distinguished from the action of an intelligent being. For instance, a computer may play chess better than any human and still not be able to read Shakespeare, which simply isn't necessary for playing chess.

The conservative society wasn't wholly grateful to one of its national war heroes. Turing was convicted of homosexual acts and accepted to undergo a chemical treatment, but couldn't bear the sentence and departed from this life.

The chemical treatment was the ultimate "scientific" finding to "cure" 32 (Turing 1950) 
ltimate "scientific" finding to "cure" homosexuality, based on nothing else than the belief that homosexuality is a disease. These "scientists" didn't understand that sexuality is pure pleasure designed to trick humans into reproduction but not limited to that, everybody can make their own choices as long as they don't harm anyone. A bit of abstraction helps to understand reality and to get out of clichés.

After World War II, the most decisive impact on mathematics had Nicolas Bourbaki, a mathematician who never existed. It was a pseudonym for a French collective of top mathematicians that aimed at writing a complete treatise of analysis and later tried to extend the volumes to all important branches of mathematics. Very much in the spirit of Hilbert, the books started from foundational axioms ${ }^{33}$, focused on generality and were written in a stark linear style, that is, everything is well founded and deduced from previous expositions. On the other hand, the authors barely gave motivating examples, they seemed to be guided by the idea that a specific example can never show a complete theory and therefore is always misleading. In their effort to be complete and self-contained, the authors avoided to step outside of their realm so that the books contain almost no applications from other disciplines. Nevertheless, because of their completeness, the books could be found in nearly all math departments; and because of their linear style, they were quite convenient for math professors to prepare their lectures.

Clearly, with the diversification of mathematics after Hilbert and the continuing intuitive style of applying mathematics in physics and other disciplines (like Poincaré), it was time that someone gathered the mathematical know-how and put it on a solid footing. Honestly, the French collective did a superb job. However, after World War II and before 33 (Bourbaki 1939) the market was flooded with more pedagogical text books, Bourbaki's perspective became the predominant style in (European) mathematics and was passed to the next generation. Moreover, because of the cold war, space race and the fear of a technological gap, both political sides granted (particle) physics and space exploration a huge amount of freedom. Mathematics, considered a basic science, benefited greatly from these developments, nobody from the treasure department asked if the money spent on mathematicians will produce any revenues. Quantum field theory, mathematically still incomplete, provided enough challenges for mathematicians. A promising way to obtain research funds was to relate somehow one's own work to quantum field theory, it didn't require to mention a nuclear bomb, any advance in nuclear physics could be the spark for the construction of a more destructive weapon.

The governments and their finance ministers, who usually don't understand much about pure mathematics, never noticed that pure mathematicians liked to ask and to solve their own questions, sometimes a little far from reality. A frequent justification was that "it might be useful in the future". This is an interesting way to give one's life a purpose but a meaningless excuse. Imagine if all these smart brains would have dedicated themselves to real-world applications, how much useful mathematics could they have produced?

Bourbarki's style of mathematics created a certain arrogance of the purest pure mathematicians toward applied mathematics. They started to believe that pure and applied mathematics are different subjects and that the applied mathematicians were inferior because they "only apply our theorems". The wake-up call came with the economic recession when research foundations did ask the question of financial benefit. Quite a few were upset 
lications in weaker journals were more successful in grant applications. In economics, this is the law of the free market, if there is no demand, there will be no need for a supply.

The reader may be warned not to stumble into the same pitfall. The question is not if pure mathematics is more powerful or if applied mathematics is more useful, the problem is that there should be no separation between applied and pure mathematics. Bourbaki's effort was correct and necessary, someone had to establish the foundations of mathematics, and of course, the so-called "applied" mathematicians will always draw inspiration from the so-called "pure" mathematics. On the other hand, all mathematicians, no matter if they consider themselves pure or applied or algebraists or analysts or geometers or topologists or whatever, should ask themselves why they are doing what they are doing and have a valuable goal in mind, which could in fact be a vision of what mathematics should achieve in the future.

If Bourbaki's goal has ever been to collect the whole mathematical knowledge in a series of books, then the collective failed already during its existence. Mathematics developed far too quickly for a handful of debating elite mathematicians to keep up with compiling all knowledge into books. At this point, it would also be too exhaustive to mention all brilliant mathematicians of that century. The easiest way to name some masterminds would be to list the Fields medalists, they all deserve to be called geniuses. To keep the paper short, I will bring up only a few exceptional ones.

The Fields Medal is often regarded as the Nobel Prize in Mathematics. There are other prestigious international awards in mathematics, for instance the Abel Prize and the Crafoord Prize were created as a complement to the Nobel Prize. What makes the Fields Medal so special is the age limit. The Fields laureates must have obtained their groundbreaking results at an age under 40 so that, unlike a 97 years old Nobel laureate, they can still shape their discipline in the long term. That this actually happens can be seen in the Citation Index. Lifetime citations of Fields medalists usually ramp up from hundreds to thousands. It's fair to say that the Fields Medal committees have now a greater impact on mathematics than Bourbaki.

It has wildly been speculated why Nobel didn't establish a prize in mathematics. $\mathrm{Ru}$ mors spread that the acclaimed mathematician Gösta Mittag-Leffler was the cause. Some say that Nobel felt an antipathy for MittagLeffler that passed on to mathematics, others say that Nobel felt betrayed by his contemporary in a love affair, even the name of the alleged woman was disseminated: Sofia Kovalevskaya. Sometimes the simplest explanation seems to be the right one. Nobel wrote in his will that the prizes should be created for those that accomplished the "greatest benefit to mankind". So, what is the benefit to mankind if some talented mathematician aged under forty proves the Riemann hypothesis? It would definitely be worth a Fields Medal and mathematicians all over the world would be delighted, but most people outside mathematics, including famous engineers and inventors, can't see much benefit to mankind in it. No one seems to be surprised that there is no Nobel Prize in Fine Arts.

What is true though is that Mittag-Leffler helped Sofia Kovalevskaya ${ }^{34}$ to get a position at Stockholm University. This made her the first confirmed female professor in mathematics and she was the first woman to earn a $\mathrm{PhD}$, too. Note that she is also the first woman mentioned in this paper, which has a sad reason. Untillate 19th century, women were not allowed to study at universities. What science has irretrievably lost by excluding half of man- 
kind from research becomes evident from her $\mathrm{PhD}$ thesis, in which she proved the main existence and uniqueness theorem in the theory of analytic partial differential equations ${ }^{34}$.

The most influential woman in mathematics was Emmy Noether, one of the first women to earn a habilitation (teaching license), which was rejected in her first attempt because of a ban for women. Her contributions to abstract algebra are outstanding and still part of all advanced courses in ring theory (objects with addition and multiplication like the integer numbers). In physics, she proved that any continuous symmetry leads to a conservation $\mathrm{law}^{35}$. This includes in particular the most important law of nature, the conservation of energy, which follows from an invariance in time translations. Thus it can be said that humanity owes the discovery of the most important law of nature to two women: Émilie du Châtelet was the first person to postulate it and Emmy Noether proved it mathematically.

Among the Fields medalists, there is only one female mathematician, Maryam Mirzakhani. Unfortunately she didn't live long enough to continue her great achievements. Her work is remotely related to the groundbreaking ideas of another Fields medalist, who stands out by his unusual personality: Alexander Grothendieck. Once more it will be shown that the behavior of a rational mathematician doesn't have to be rational.

Grothendieck had a difficult childhood. His father was a Jew, anarchist, a migrant from the Soviet Union and illegal in Germany, so there was hardly another combination that would have triggered more hate from the Nazis, nomatter what a niceperson hemight have been. Because of the imminent threat of the Nazis, the family fled to France, but was captured there, and Alexander's father was one of the first to be killed in a concentration camp. Alexander and his mother survived the Nazi regime.
After World War II, he first studied mathematics at the provincial University of Montpellier, later at the more prestigious École normale supérieure in Paris, and then specialized in functional analysis in Nancy, working with Fields medalist Laurent Schwartz and Bourbaki cofounder Jean Dieudonné. He soon became a rising star in functional analysis, studying the scary subject of topological tensor products, where different completions yield different algebras that are difficult to classify. Grothendieck solved the analysis problem with abstract algebraic methods ${ }^{36}$. What a luck for mathematics that this algebra genius was driven into functional analysis! Later he laid the algebraic foundations of the Atiyah-Singer index theorem, mentioned previously as one of the most celebrated theorems of the 20th century.

Knowing his childhood, it is no surprise that Grothendieck was a pacifist. To avoid military service, he kept his stateless refugee status, traveled to Brazil and the US, and finally ended up in Paris again. There, at the Institut des hautes Études scientifiques (IHÉS), he found the perfect research environment as Paris turned into the center of algebraic geometry. That his talent was widely recognized can be seen in the fact that he became a member of the Bourbaki collective, the ultimate step of his rise from a provincial math student to the top elite circle of French mathematicians.

Grothendieck's research was substantially driven by the attempt to prove the Weil conjectures ${ }^{37}$. The Weil conjectures arise from the interest of counting solutions of algebraic expressions, where the solutions belong to a finite set of numbers. Here, numbers mean a set that allows to perform the operations addition, subtraction, multiplication and division. An example is the set $\{0,1\}$ with the usual operations and the condition $1+1=0$. So it is not true in mathematics that $1+1$ is always 2, the number 2 might simply not be 
part of the game. This is actually exactly the way in which a single bit in a computer performs its computations. Such numbers and solutions of algebraic expressions have for instance an application in coding theory. André Weil associated to the counting of solutions a function that resembles the Riemann zeta function which gives rise to Riemann's hypothesis.

Starting from a few examples, Weil made four conjectures about this function that became Grothendieck's main motivation for studying algebraic geometry.

Although Grothendieck proved only parts of the conjectures and didn't succeed in proving all of them, his work on the Weil conjectures shows clearly his extraordinary style of thinking. Grothendieck was a true master of abstraction. When he tried to solve a problem, he started to understand it in an abstract manner, discovering in that way new questions. If he couldn't find a solution, he passed to more abstraction to understand the true nature of the problem, and then to more abstraction to unify different theories, and then to more abstraction to reveal different aspects of the problem as parts of the same whole. At the end, his final goal was not the proof of the conjectures but to establish the right framework in which the conjectures could be proven naturally. Grothendieck didn't seek fast fame by solving these difficult mathematical problems, rather the contrary, the proof of these conjectures should be the ultimate evidence that he revealed the correct underlying principles. When his student and now Fields medalist Pierre Deligne showed him the proof of the final and hardest part of the conjectures ${ }^{38}$, Grothendieck was evidently disappointed by the "trick" that Deligne had been using. Manybelieved thatGrothendieckfeltenvy toward his former student but I don't think so. I believe that Grothendieck was honestly disappointed that the proof didn't emerge from his foundational theories, so he may have felt in that moment that his mathematical endeavor will remain unfinished.

His way of thinking shaped a mathematical discipline that mathematicians call category theory. Category theory is a form of abstraction that reduces everything to its essential structures and adds then step-by-step more properties. Grothendieck was so fond of this theory that he wanted to lay the foundations of mathematics completely in category theory, as much as Bourbaki wanted to lay the foundations of mathematics in set theory, obviously quite a different way of thinking.

Absolutely deserved, Grothendieck was proposed to receive the Fields Medal in 1966 at the International Congress of Mathematicians in Moscow. It is an irony of fate that the congress was held in the country from which his father escaped because of his anarchist ideas. Having strong political views similar to his father, it is no surprise that Grothendieck refused to travel to Moscow.

Shortly after receiving the Fields Medal (in absence), the decline of his mathematical career started. Opposing actively the Vietnam War and being caught up in the 1968 student revolts, he found a new obsession in political activism. Grothendieck launched a political group called Survivre, a movement fighting for the survival of all life threatened by the destruction of the environment and militarism. He tried to attract fellow mathematicians and senior colleagues, but was mostly met with incomprehension. When Grothendieck learned that the IHÉS received military funding, he quit his job at the IHÉS and, after some temporary positions, returned to the University of Montpellier, the same provincial university where he once came from. His social life deteriorated even further, he withdrew from mathematics, cut ties with family members and friends, and re- 
treated from public life to a small village in the Pyrenees. There he spent his final years like a reclusive monk dedicating himself to religion (e.g. Buddhism), meditation, spirituality and introspection.

His last years were only disturbed by intrusive admirers with dubious motivations. I can't imagine that a perfect stranger knocks on the door of a Nobel Prize winner saying "Madame, you are famous, I would like to talk with you about chemistry!"

Much has been speculated about his reasons to withdraw from mathematics, and his post-mathematical life has attracted a lot of curiosity. Grothendieck wasn't completely silent in all these years, rather the contrary, he wrote letters to colleagues and thousands of pages of "Meditations" to explain himself in a poetic style that is a bit difficult to follow and often leads to nowhere. His most famous work in this time is Récoltes et Semaille, which many regard as a personal judgment about mathematics expressing dissatisfaction and resentments. This seems not to be true, although he elucidates his disappointments of a "certain sort" of colleagues, he writes quite positively about his "elders" and friends in mathematics. Récoltes et Semailles appears to be an attempt to reflect about his own life as a mathematician, to illuminate his passion for knowledge, and to share his visions for mathematics.

Instead of reading thousand pages of reflections, there is a faster way of understanding Grothendieck. In 1988, Grothendieck was to be awarded the prestigious Crafoord Prize which, as mentioned above, is considered a complement of the Nobel Prize. Grothendieck refused the award and explained his reasons in a letter sent to twelve scientific journals ${ }^{39}$. Apart from making clear that he doesn't need more material wealth and that the money could be used better for those in need, he did in fact complain about a decline of ethics and values in the 39 (Grothendieck 1989) scientific "milieu".

So, what might he have been complaining about? There are things happening in mathematics that don't meet Grothendieck's high ethical standards. Grothendieck mentioned explicitly theft among colleagues, he probably was upset that some ideas from his seminar at IHÉS surfaced in mathematical journals. He also didn't like the elite thinking at the renowned research centers in Paris and in the Bourbaki collective. Grothendieck emerged from a precarious social background and felt that he had to prove his worth to be accepted in the elite circle that showed a certain arrogance toward those who don't reach the highest levels. In Récoltes et Semailles, he complained about egocentric characters. Of course it hurt him that colleagues and supposed friends publicly commented without comprehension on his spiritual path, but he was also annoyed by those who despise the "soul". This can be translated in a lack of vision and passion for mathematics, all that counts is the number of publications and in which journals. Abstraction became a means for the production of "results" without any motivation, just to define another area in which one can harvest a few new theorems. Known theorems are rewritten in a more abstract or generalized manner to claim an original result. If a theorem is too difficult to prove, the necessary conditions are stated in a definition in order to avoid hard work in the proof. The publications of friends are excessively cited so that the chances are high that the unaware editor sends the manuscript to a "specialist in the field" who certainly accepts the paper. In fact, the high number of mathematical papers that will be forgotten in the future is an obstacle for the progress of mathematics rather than an advance. Mathematics is getting too dispersed to generate profound impact by collective efforts, too much time is wasted by proving 
useless conclusions. Competition, not o nly for prizes and rewards, started to dominate mathematical research although collaborative work for a common goal would move mathematics forward at a faster pace.

However, Grothendieck's refusing letter contains another hint at the true reasons for his withdrawal from society. He writes about an "unprecedented civilization collapse". This is a typical, irrational, catastrophic thinking of people with an increased anxiety in a crisis situation. The withdrawal from society on the one hand and the aggressive tone of the letter on the other indicate "flight or fight" responses to stressful events. Writing thousands of pages to explain oneself to the world from which one has retired is quite the contrary of finding inner peace, it was done with the same obsession as his mathematical research. Such symptoms can be found in a manual of mental disorders. I am not making a diagnosis and I am not able to do so. All I want to say is that, instead of trying to find an explanation for irrational behavior, friends and acquaintances should think about if a person close to them needs professional help.

The next story belongs to the present century but resembles a lot the last one, therefore I will tell it now. There is a mathematician who did not only reject the Fields Medal but also the Clay Millennium Prize of one million dollars. Up to date, only one of the seven Millennium Prize Problems has been solved. The genius, who did it, is Grigori Perelman and the problem was the above mentioned Poincaré conjecture. A striking similarity of the two stories is that both complained about a decline of ethical standards. Yet there is a huge difference between the two cases: Perelman's behavior was always consistent. Of course the media went crazy and journalists made big fuss about it. What these ladies and gentlemen don't understand is that not everybody has the same ethical standards, not everybody has the same needs, not everybody feels justice the same way, and not for everybody one million dollars have the same value.

There might be someone who prefers being who he is over being a millionaire. Grigori Perelman hasn't had many friends and didn't spend much money before he got famous. He did what he liked most, venturing in difficult mathematics, a world of its own. He had what he needed, much like Grothendieck who lived his final years from a small pension. Why should more money and more publicity make him more happy? To all admirers, please don't knock on his door shouting "Súdar', you are famous, I would like to talk with you about mathematics!"

Perelman proved the Poincaré conjecture by using a technique developed by Richard Hamilton in the 1980's called Ricci flow ${ }^{40}$. Imagine that your beautiful spherical car has crashed. What the Ricci flow does is to smoothing out the deformations back to a round shape like a mechanic. But if the mechanic doesn't work carefully, he may create new dents and spikes known as singularities in mathematics. Richard Hamilton realized that his tools may be used to prove the Poincaré conjecture but he was not able to deal with the singularities. Perelman had a background in Alexandrov spaces, which behave fairly more singular than round spheres, and found a way how to deal with the singularities. He proved that some kind of singularities don't even occur, and others can be removed by cutting and paste techniques. Perelman was totally aware of what his findings mean - the first solution of a Millennium Prize Problem, thus he published it on a preprint server ${ }^{41}$ and sent copies to several specialists in the field so that the proof can publicly be verified instead of an anonymous peer review. As Perelman said in an interview: "If the proof is correct, then 
no other recognition is needed." However, not sending it to a peer-review journal meant not fulfilling the conditions of the Millennium Prize Problems so that a mathematician with high ambitions and lower ethical standards might consider the race as still open.

One of the mathematicians, who received directly a copy from Perelman, was Fields medalist Shing-Tung Yau. He received the Fields Medal for proving e.g. the Calabi conjecture 42 , which later became important in string theory because it allows to decide when a manifold can be used to compactify the extra spatial dimensions in string theory. These manifolds are now known as Calabi-Yau manifolds.

At the turn of the century, his glory had already faded and he acquired new fame as someone who meddles in disputes and politics. Hamilton was closely related to his research group and Yau very likely saw the possibility of proving the Poincaré conjecture by using Hamilton's techniques. It might have felt worse than a fourth position in the Olympic Games to see that some no-name finished the proof, but nobody should be surprised that suspicions were aroused when two of his former students published a paper about a complete proof of the Poincaré conjecture ${ }^{43}$ in the Asian Journal of Mathematics, where Yau is coincidentally one of the two chief editors. No serious referee could have ignored Perelman's work and the suspicions were substantiated when the authors had to publish an Erratum ${ }^{44}$ in which they admitted that large part of their arguments were already obtained by Perelman. Now Perelman had to make a choice, either to win one million dollars and to be part of this circus of disputes, accusations, dishonesty and low ethical standards, or to disappear in order to keep his honesty and high ethical standards intact. To keep his inner peace, he had to choose the

\footnotetext{
42(Yau 1977)

43 (Cao and Zhu, A Complete Proof [...], 2006)

${ }^{45}$ (Wiles 1995)
}

second.

I would like to mention the story of a mathematician who deserved the Fields Medal and didn't get it. Of course, there are many of them, but one narrowly missed the medal because of the age restriction. Slightly below the age limit, Andrew Wiles submitted a paper on elliptic curves ${ }^{45}$ that implied the proof of a very famous problem that is astonishingly simple to state but resisted a proof for more than 350 years. The problem is known as Fermat's Last Theorem and conjectured that the equation $a^{n}+b^{n}=c^{n}$ does not have solutions for positive integers $a, b$ and $c$ if $n>2$. Euclid knew already that the case $n=2$ has infinite many solutions (Pythagorean triples). Fermat may not have had such strong ethics as Perelman because he wrote in the margin of a book that the equation doesn't admit solutions for $n>2$ and that he found a marvelous proof which is unfortunately too long write in the margin. His remark probably put a few mathematicians in the wrong direction because it seems that an elementary proof does not exist.

Wiles proved the theorem with heavy machinery from Grothendieck's algebraic geometry, and by combining the theory of elliptic curves with something that has to do with (weighted) invariance properties, a subject that can easily become complicated. In the theory of elliptic curves, elliptic curves do not look like ellipses, rather like a donut. The name comes from the fact that these functions arise as inverses of elliptic integrals, which appear in the calculations of perimeters and areas of ellipses and ellipsoids. No known elementary function solves these integrals, thus they are interesting in their own right and intensely studied. Studying the inverse functions is alike to a dual approach. Wherever there is mathematics, there is a dual structure, the cleverest mathematicians know how 44 (Cao and Zhu, Erratum, 2006) 
to use it. The surprise in this example is that the inverse function can be studied in the complex plane (I wrote already about the fabulous properties of this number system), there they behave very well - as if they were defined on a donut.

If someone writes down all the additional knowledge accumulated after Fermat's death that is necessary to prove Fermat's Last Theorem with Wiles' methods, then the exposition will easily exceed thousand pages, obviously a bit more than what would fit in the margin of a book. Wiles worked six years on the proof and didn't publish much in this period, actually so little that he would have felt the consequences at a university or in a country with a rigid evolution system, deeming his attempt as a failure before its completion. Unfortunately, the initial proof contained an error, so that Wiles had to work another year to fill the gap and passed the age limit for the Fields Medal. The International Mathematical Union, which is in charge of awarding the Fields Medal, honored these achievements with the first and only silver plaque.

The only physicist to receive the Fields Medal is Edward Witten. He is best known for his work in string theory, quantum gravity and supersymmetry, in short, the theory of everything. Physics has a problem with unifying quantum field theory - the theory of eleymentary particles and their electromagnetic, strong and weak interactions - and gravity, commonly known as the relatively weak attractive force between masses but mathematically more precisely Einstein's theory explaining how energy warps our space-time. The puzzle is why gravity behaves so differently than the other inter-actions. Whoever solves the puzzle is a contender for the Nobel Prize.

String theory is (was?) a candidate for the theory of everything. The basic idea is quite natural, unless otherwise proven, there is no reason to assume that elementary particles are point-like, all this wave function business hints otherwise. Mathematically, the next difficulty is to step up one dimension, i.e. from 0-dimensional particles to 1-dimensional vibrating strings, then to a 2-dimensional membranes (2-branes) and, more generally, to $p$-branes, where $p$ denotes the dimension.

Curiously, string theories are only consistent in higher dimensional space-times, for instance in 26 dimensions, 10 dimensions or, in the case of Witten's unifying theory, $11 \mathrm{di}$ mensions ${ }^{46}$. The fact that our senses perceive exactly three spacial dimensions is not a proof that the elementary particles live only in three spacial dimensions. Actually the mysterious dark matter and dark energy could be effects from higher and lower dimensions. The issue is what to do with the extra dimensions. Witten et al. ${ }^{47}$ proposed to compactify them with manifolds that have the right properties like being "very small", not breaking the considered symmetries and being Ricci flat so that they don't produce gravitational effects. Examples of manifolds that satisfy these conditions are the above-mentioned Calabi-Yau manifolds.

Once the mathematicians got to know the importance of Calabi-Yau manifolds in string theory, there was no stopping the research of Calabi-Yau properties. Suddenly generalized Calabi-Yau manifold appeared, Calabi-Yau algebras, Calabi-Yau categories, and so on, each time a bit further away from the original motivation. Mathematically it is justified, mathematicians want to understand and to classify these structures, or any other. String theory had a huge impact on several mathematical research areas like cobordism, 4-manifolds, differential geometric invariants, moduli spaces, Chern-Simons theory, knot theory, to name a few.

Nevertheless, being a physical theory, it

\footnotetext{
${ }^{46}$ (Witten 1995)

${ }^{47}$ (Witten et al. 1985)
} 
is also justified to ask the question if string theory has anything to do with reality. Unfortunately, no experiment has ever proven string theory to be a valid model. On the other hand, there is so much freedom and there are so many parameters in string theory that it might be possible to design a mathematical model that matches known experiments without describing the actual physical reality. (Maybe the extra dimensions don't exist?) String theory is one of the most advisable examples to ask the question when is the best time to stop research on a theory that doesn't produce verifiable results. After fifty years? Hundred? When a better theory is found? My prediction is that disciples of leading string theorist will continue to study string theory, and their disciples too.

Another Fields medalist, who created an exotic theory about the nature of reality, is Alain Connes. In his PhD thesis, he discovered time, that is, the unique (up to inner automorphisms) 1-parameter group leading to equilibrium states in quantum statistical mechanics ${ }^{48}$. Then he got the Fields Medal for the solution of a difficult classification problem of operator algebras ${ }^{49}$, the sort of operator algebras that build up algebraic quantum field theory. After receiving the award, he used his freedom at the famous Field medalist producing research institute IHÉS to create a completely new mathematical theory: noncommutative geometry ${ }^{50}$.

The motivation for noncommutative geometry is quite convincing. Classical mechanics and general relativity have a purely geometric formulation in terms of symplectic and pseudo-Riemannian manifolds, respectively. On the other hand, quantum physics deals inherently with noncommutative operators. For many years, mathematicians and physicists tried to deduce quantum mechanics from classical mechanics although the opposite way would be more natural, namely to view classical mechanics as a many-particle-limit of the quantum behavior of the constituent particles. If the classical theories are of geometric nature, so should be the noncommutative operator algebras in quantum theory.

Connes and some physicists went even further by considering the possibility of a noncommutative space-time at the very tiny Planck length scale. The reason comes from a measurement problem. If one wants to determine a point in space, one has to perform a measurement to "see" the point, and the only possibility is a scattering-type experiment with a particle of non-zero mass; photons always move too fast, i.e. arrive from a different place. To measure the point more and more precisely, more and more mass (or energy, which is the same - says Einstein) has to be concentrated in a smaller and smaller region, therefore creating eventually a micro black hole, that's what Einstein's theory predicts. But a black hole is characterized by an event horizon beyond which no information reaches an observer. The theoretical minimum radius, which does not depend on the energy, is known as the Planck length. So, what is the point of considering a point in space that can never be measured below Planck length? It is pointless to speak of the existence of something that can never be observed.

Connes proposed not to study the points but the functions defined on such (quantum) spaces, for instance the coordinate functions. This matches exactly the idea of algebraic geometry, in fact, Grothendieck already proposed a topos category which avoids completely the notion of a point in a topological space (= space with a concept of continuity), though without a physical motivation. Heisenberg's uncertainty principle teaches us that there is a minimum volume in 2-dimensional quan-

\footnotetext{
${ }^{48}$ (Connes 1973)

${ }^{49}$ (Connes 1976)

${ }^{50}$ (Connes 1994)
} 
tum phase space, exactly the same effect can be obtained by considering noncommutative space-time variables.

As appealing as this idea might be, the problem is again the experimental verification. Only if certain quantum states can be maintained stable over a long period of time, there exists a chance of measuring noncommutative space-time effects. On the other hand, it is already clear that this theory will never be useful for creating more powerful power stations nor more deadly weapons of mass destruction since we are talking about something at a very, very tiny scale. So, why bother at all? One reason was the hope to avoid the infinities in the above mentioned Dyson series. Other reasons are more of mathematical nature. There are (topological) spaces, like foliations and Penrose tilings, along with physical topological insulators, that behave badly when studied with conventional methods but possess a rich noncommutative geometry. It is no surprise that Witten incorporated these structures in string theory ${ }^{51}$.

Even if all hopes of describing a noncommutative space-time fail, it seems math ematically worth to study noncommutative geometry because it is not a specialization but a unifying generalization. It incorporates under the same framework a wide range of different disciplines like algebraic geometry, differential geometry, differential operators, operator algebras, topology, algebraic topology, Lie groups, representation theory, spectral theory, invariant theory, index theorems, etc., and generalizes them to a possible quantum world. At least, in this way, one can learn a lot about mathematics. Moreover, mathematics works best when many disciplines intersect.

Finally let me mention the most prolific mathematician of the last century, working in number theory and discrete mathematics (among others): Paul Erdős. Number theory 51 (Seiberg and Witten 1999) accompanies mathematics since the beginning, and prior to the digital age, it was regarded by many mathematicians as the high art of mathematics but completely useless, i.e. without practical applications. Now it is indispensable in computer science and cryptography. Again one might ask the question when to stop the research on a useless theory. After fifty years? Hundred? Immediately, until an application demands research on it? Although number theory is a good example that it would have been a bad decision to abandon research with future applications, I personally consider it a bad habit of mathematicians to justify their work with the words "it might turn out useful in the future". It might also be a waste of resources for fruitless theories.

Erdös is not known for establishing a new theory, but as one of the greatest problem solvers. He wrote more than thousand papers and had several hundreds of collaborators who he found by constantly traveling around the world and living an eccentric, yet spartan lifestyle dedicated solely to mathematics. It occurred to someone to define the Erdo"s number as the collaboration distance to Paul Erdős. So, direct collaborators have Erdős number 1, and a collaborator of a collaborator who collaborated with a collaborator of Erdös has Erdős number 3. It is amazing to see that nearly all mathematicians with a couple of coauthors have a (finite) Erdös number and almost all of them have a number less than 8 . This shows that mathematicians are well connected and refutes the image of mathematicians as hermits solving problems behind closed doors.

In conclusion, what history shows us is that mathematics and other sciences were strongly influenced by individual persons called "geniuses" (in a simplistic view, some geniuses don't like to be called genius). These characters with groundbreaking results and an in- 
credible impact on human progress can be anybody and anything: men, women, migrants, conservatives, conformists, rebellious, perfectionist, intuitionist, innovative, creative, egoistic, egocentric, eccentric, ascetic, with high ethical standards, with low ethical standards, homosexual, with mental disorders, blind - not to mention all the clichés of mathematicians that are fed by some protagonists.

Needless to say that there are many more geniuses who were not mentioned here, and even more who can abstain from being called so. Each year, a huge number of research papers are published which will be completely forgotten in a few decades. Can't we leave mathematics in the hands of the top elite masterminds, like many radio stations that play always the same songs of a few famous ones from the billboard charts, instead of cluttering libraries and webpages with useless publications? Actually, a collective effort of problem solving, e.g. on webpages specially designed for this purpose, could lead to a much faster progress in mathematics and other sciences than a narrow-minded competitive behavior (Who is the best? Who writes more papers?), since numerous persons generate more new ideas on a specific issue than a single human. On the other hand, the theory of the free market tells us that competition always leads to progress.

The question needs no answer because it won't change in the near future. However, the answer to the question if it is worth that so many mathematicians explore mathematics is a completely different one. First, mathematics trains people in a particular way of thinking: abstract, logic and deductive. A society that produces a great amount of mathematicians has many people available for tasks that involve abstract, logical and deductive thinking. Only if the environment is not fruitful enough, mathematicians become useless. Furthermore, nobody knows if the next genius comes from London or Morelia, so the stage should be set in both places. I can also see a benefit in the production of the countless, soon forgotten publications. They serve as a resonance of the top research. Those areas with the most vibrant research activities will survive, others will slowly decline.

Looking at the history, it can be seen that progress was mostly inspired by applications. Working solely in potentially useless theories seems to be a recent phenomenon, although there are also examples like number theory that were considered useless for centuries but found a late application. However, nobody should take those examples as a cheap excuse to do whatever (s)he wants to do without ever asking the question what the motivations are for doing it. If it is not a direct application, it might be a vision of what mathematics should achieve in the future.

Anyway, a trend can be observed that governments and research funds prefer applications over purely theoretical research. This is perfectly understandable. Why should a sponsor not ask what to get back from the money? Pure mathematicians complain about it, they see the future of pure mathematics threatened. Having said that, I can't see the problem. If mathematicians focus on concrete problems, they will derive new, powerful, abstract theorems, as some of their heroes did in the past. It's the division between pure and applied mathematics, which I regard as a problem. Now, if this division persists, what is the future of pure mathematics?

Today, September 19, 2021, I can predict the future of pure mathematics with absolute certainty: It will be an evolutionary process. As long as pure mathematics has a benefit for the society, it will survive. For instance, if pure mathematicians produce highly ca- 
pacitated teachers at all educational levels, or qualified innovators with unusual ideas in the private sector. There will surely be a decline in research funds allocated to pure mathematics, which pushes more and more researchers into applied math. Nevertheless, the greatest geniuses are free spirits that cannot be caged, they will always revolutionize science without asking if the society calls it pure or applied.

\section{The Present}

\section{The Future}

The past has seen many scientific revolutions, often initiated by individual persons but always carried on by collective effort. We don't have to wait for the next big shot, the next revolution has already started!

The future will be revolutionized by Artificial Intelligence and Machine Learning, as much as computers and the internet revolutionized our daily life. It is the first time in the history of mankind that machines are able to learn much faster than humans; it is the first time in the history of mankind that computers develop something that may be called intelligence, each time faster than humans, and finally they will teach themselves. This is the beginning of an exponential growth! Better machines will train future machines to become better, knowledge and capabilities will increase at an astonishing speed. Computers don't need to be punished by death if they make mistakes so that natural selection evolves advantageously, computers can be restarted and can learn from their mistakes again and again.

It doesn't matter if these machines are good at a single task, we can build many of them for different jobs. What matters is that they perform their tasks better than human minds.
Wherever there are strict rules, like chess, data analysis or mathematics, machines will outperform our brains because brains don't evolve so fast. The computer program AlphaGo Zero learnt to play Go by playing against itself and not only surpassed all professional human players in a few days, but also all previous versions, accumulating more knowledge than human mankind in thousands of years ${ }^{52}$. No human will ever again become world champion in Go.

Go is just a game, but multi-resistant bacteria are a serious threat to our survival. Last year, a team from MIT let machines analyze data from known antibiotics and millions of molecule structures ${ }^{53}$, an impossible task for a crew of biologists and chemists. The algorithm did not only identify powerful new kinds of antibiotics, it also found one against a strain previously considered untreatable. Similar to Go players, researchers were stuck in their beliefs which molecules would be the correct ones and "discovered" always the same structures. The machine had no beliefs, it learnt everything from scratch and did moves no one else has done before.

The next task is to analyze the human DNA, obviously too much information for our brains. In computational biology, "world championships" of protein structure prediction are organized among more than 100 participating research groups. The goal of these "games" is to identify protein structures from amino acid sequences. It doesn't need much fantasy to understand its potential use in medical drug design and in the creation of new enzymes in bio-engineering. Artificial Intelligence also assists already the inverse process, namely protein design, evidently with the same potential applications. Last year's winner was Google subsidiary Deepmind, the same research laboratory that created the world champion in Go ${ }^{54}$. No one should 
seriously be surprised that the world champions come from a private company with a huge amount of money and salaries higher than those of university professors. With the actual (public) university funding, the race is already lost to the big tech companies and an authoritarian regime.

I am not pretending that there is no danger although Artificial Intelligence doesn't have free will. Bad people can show them bad things, I don't think I need to explain it further. Machines are as "good" as we make them, and as good and as bad as us - their creators. Imagine that a machine receives randomly data from the internet, how much garbage will it learn? Hopefully nobody will be so stupid to feed them with piles of fake news, but someone was already so intelligent to let them learn from criminal statistics in the US for the creation of suspect profiles. Now it's too late, now they are already as biased as middle-aged, white, male police officers exactly as those who collected the data.

If pure mathematics turned out one day to be the most powerful science, machines could be taught to read math articles, all of them, also the long ago forgotten, so take care, someone may find a mistake. Maybe they process only very carefully selected ones, it doesn't matter, they will definitely be able to store the exact content of many more research results than a human can read and understand in his life. The first step serves just for storing information, just to accumulate knowledge without being able to use it. In parallel, they could learn from this information, taught by a human or another machine. Then they are prepared for the search of new connections, first randomly, but since the beginning directed. Finally they will be capable of finding new theorems, but contrary to humans, none will ever be forgotten again, all data will be saved for centuries, and they can accumula- te more and more theorems at a faster pace than humans, no human will be able to challenge them. If they find a connection that they can't prove or disprove, like Riemann's hypothesis, someone may show them to call it a conjecture.

As alluded in the first line of the previous paragraph, this fictional scenario only happens when the benefit justifies the effort. If there is no (financial) reward in finding the best match between an 11-dimensional string theory and all known parameters and outcomes of measurements, nobody will start such an enterprise. However, what has already started and will gain a momentum that surpasses human comprehension is the search for new and more efficient algorithms in data analysis.

A country not investing in Artificial Intelligence and Machine Learning will be left behind like a country not investing in computers and internet technologies. If someone in charge reads this, I wish her/him to make the right decisions and good luck!

\section{Acknowledgement}

I am very grateful to the referees for the careful reading of the manuscript.

\section{References}

[o] All additional information was found on THE INTERNET.

[1] WiKIPEDIA. HTTPS://WWW.WIKIPEDIA.ORG.

[2] London Institute FOR MATHematical Sciences.” httpit//Lims. AC.UK/ 23 - MATHEMATICAL-CHALLENGES/.

[3] Clay Institute of Mathematics." https:// WWW.CLAYMATH.ORG/MILLENNIUM-PROBLEMS.

[4] DEEPMIND. HTTPS://DEEPMIND.COM/BLOG/ ARTICLE/ALPHAGO-ZERO-STARTING-SCRATCH.

[5] NatURE. HTtPs://WWW.NATURE.COM/ARTICLES/ D41586-020-00018-3 
Abel, N. H. MÉmoire SUR les ÉQUATIONS ALGÉBRIQUES OÙ ON DÉMONTRE L'IMPOSSIBILITÉ DE LA RÉSOLUTION DE L'ÉQUATION GÉNÉRALE DU CINQUIÈME DEgRÉ. CHRISTIANIA: DE L'IMPRIMERIE DE Groendahl, 1824.

Atiyah, M. F., And I. M. Singer. “The Index of Elliptic Operators I." ANNAls OF MATHEMATICS, 1968: 484-530.

Benzmüller, C., And B. Woltzenlogel-Paleo. “Automating Gödel's Ontological Proof of God's Existence with Higher-order Automated Theorem Provers." Proc. European Conference on Artificial Intelligence 263 (2014): 93-98.

BoltzMAnN, L. "ÜBer DIE BEZIEHUNG ZWISCHEN DEM ZWEITEN HAUPTSATZ DER MECHA-NISCHEN WÄRMETHEORIE UND DER WAHRSCHEINLICHKEITSRECHNUNG RESPEKTIVE DEN SÄTZEN ÜBER DAS WÄrMEGLEICHGEWICHT.” SITZUNGSBER. D. K. AKad. Der Wissenschaften ZU Wien II 76 (1877): 373-435.

Bolyai, J. “ApPENDiX SCIENTIAM SPATII AbSOlUte VERAM EXHIBENS; A VERITATE AUT FALSITATE AXIOMATIS XI EUCLIDEI, A PRIORI HAUD UNQUAM." IN TENTAMEN JUVENTUTEM STUdiOSAM IN ELEMENTA MATHESEOS PURAE, ELEMENTARIS AC SUBLIMIORIS, METHODO INTUITIVA, EVIDENTIAQUE HUIC PROPRIA, INTRODUCENDI, BY F. BOLYAI. TyPIS CoLLEGII REFORMATORUM PER JOSEPHUM ET SimEONEM KALI DE FELSÖ VIST, 1832.

Bourbaki, N. ÉLÉments De Mathématique - Theorie Des Ensembles. Paris: Hermann, 1939.

Cao, H.-D., and Xi-P. Zhu. "Erratum to "A Complete Proof of the Poincaré and Geometrization Conjectures - Application of the HaMilton-PERElman Theory of The Ricci Flow'”' Asian Journal of Mathematics 10 (2006): 663-664.

Cartan, E. Sur la structure des groupes de TRANSFORMATIONS FINIS ET CONTINUS. VOL. 826 dE Thèses. PARIS: Université Faculté des SCIENCES, 1894.

Connes, A. "Une Classification des facteurs de TyPe III.” AnNAles SCIENTIFIQUES DE L'É.N.S. 6 (1973): 133-252.

Connes, A. "Classification of injective factors." AnNals of Mathematics 104, (1976).

Connes, A. Noncommutative Geometry. BosTON:ACADEMic Press, 1994.

De Broglie, L. "Recherches sur la théorie Des Quanta.” (Annales De Physique) 10 (1925):
22-128.

Deligne, P. “La conjecture de Weil. I." PublicaTiONS MAThÉmatiques DE L'IHÉS 43 (1974): 273-307.

Dyson, F. J. "Divergence of Perturbation Theory in Quantum Electrodynamics.” Physical ReVIEW 85 (1952): 631-632.

Dyson, F. J. “The Radiation Theories of TomonaGa, Schwinger, and Feynman." Physical ReVIEWS 75 (1949): 486-502.

Einstein, A. "Über EINEN DIE ERzeugung UND VERWANDLUNG DES LiCHTES BETREFFENDEN HEURistischen Gesichtspunkt." Annalen der PhySIK 17 (1905): 132-148.

Einstein, A. “Zur Elektrodynamik bewegter KörPER.” ANNALEN DER PHYSIK 17 (1905): 891-921.

Einstein, A. "Die Grundlagen der allgemeinen Relativitätstheorie.” AnNalen der Physik 49 (1916): 769-822.

Feynman, R. P. “The Space-Time Approach to Quantum Electrodynamics.” Physical Review 76 (1949): 769-789.

Hamilton, R. S. “Three-MANifolds With Positive Ricci curvature." Journal Differential GeoMETRY 17 (1982): 255-306.

Heisenberg, W., M. Born and P. Jordan. "Zur Quantenmechanik II." Zeitschrift Für Physik 35 (1926): 557-615.

Hilbert, D. Mathematische Probleme. Vol. 3, in Mathematisch-Physikalische Klasse, 253297. GÖtTINGEN: NACHRICHTEN VON DER KÖNIGLICHE GESEllschaft DER Wissenschaften, 1900.

Hilbert, D. Die Grundlagen der Physik. Vol. 2, in Mathematisch-Physikalische Klasse, 396407. Göttingen: Nachrichten von DeR KÖNIGLichen Gesellschaft DER Wissenschaften, 1915.

Kovalevskaya, S. "Zur Theorie der partiellen DifFERENTIALgLEICHUNGEN." JOURNAL FÜR DIE REINE UND ANGEWANDTE MATHEMATIK 80 (1875): 1-32.

LobACHEVSKy, N. “GÉOMÉtrie IMAgINAIRE." JouRNAL FÜR DIE REINE UND ANGEWANDTE MATHEMATIK 17 (1837): 295-320.

Noether, E. "Invariante VAriationsprobleme." In Mathematisch-Physikalische Klasse, 235257. Göttingen: Nachrichten von Der GeSELLSCHAFT DER WISSENSCHAFTEN, 1918.

Planck, M. “Ueber das Gesetz der EnergieverTEILUNG IM Normalspectrum.” ANNALEN DER 
Physik 309 (1901): 553-563.

Perelman, G. "The Entropy Formula for the Poincaré, H. "SUR LE PROBLÈME DES TROIS CORPS ET LES ÉQuations de la dynamique." ACta MATHEMATICA, 1890: 1-270.

Riemann, B. Ueber die Hypothesen, welche Der Geometrie zu Grunde liegen. Vol. 13, in Abhandlungen der Königlichen GesellSCHAFT DER WISSENSCHAFTEN ZU GÖTTINGEN, 133-150. Göttingen: Dieterich'sche VerlagsBUCHHANDLUNG, 1868.

Seiberg, N. And Witten, E. "String theory and NONCOMMUTATIVE GEOMETRY. " JOURNAL OF High Energy Physics 9 (1999): 032.

Schrödinger, E. "Quantisierung als EigenwertProblem." ANNALEN Der Physik 79 (1926): 361-376.

Turing, A. "Computing Machinery and IntelliGENCE." MIND 59 (1950): 433-460.

von Neumann, J. "Die Eindeutigkeit der SchröDingerschen Operatoren.” Mathema-tische ANNALEN 104 (1931): 570-578.

von Neumann, J. Mathematische Grundlagen Der Quantenmechanik. Berlin: J. Springer, 1932.

Weil, A. "Numbers of SOlutions of equations in FINITE FIELDS." BULLETIN OF THE AMS 55 (1949): 497-508.

Weyl, H. Symmetry. Princeton: University Press, 1952.

Wiles, A. "Modular elliptic curves and Fermat's Last Theorem." Annals of Mathematics 141 (1995): 443-551.

Witten, E., P. Candelas, G. T. Horowitz and A. Strominger, A. 1985. "VACuUm Configurations for Superstrings." Nuclear Physics B 258, 46-74.

Witten, E. "String theory dynamics in various dimensions." Nuclear Physics B. 443 (1995): 85-126.

Yau, S.-T. "CAlabi's Conjecture and SOME NEW RESULTS IN ALGEBRAIC GEOMETRY." PNAS 74 (1977): 1798-1799. 